\title{
MODELLING DECISIONS UNDER UNCERTAINTY IN A BEHAVIOURAL QUEUING SYSTEM
}

\author{
Carlos A. Delgado A. and Ann van Ackere \\ HEC, School of Business and Economics \\ University of Lausanne \\ Dorigny, 1015-Lausanne, Switzerland \\ E-mail: carlos.delgado@unil.ch, \\ ann.vanackere@unil.ch
}

\section{KEYWORDS}

Queuing problems, agent based simulation, cellular automata, adaptive expectations, uncertainty.

\begin{abstract}
In this paper we use an agent-based modelling and simulation approach to model a queuing system with autonomous customers who routinely choose a facility for service. We propose a Cellular Automata model to represent the customers' interactions and study how customers use their own experience and that of their neighbours in order to update their memory and decide what facility to join the next period. We use exponential smoothing to update the customers' expected sojourn time. We incorporate uncertainty regarding these expectations into the customers' decision. We compare the resulting behaviour when customers take into account uncertainty to the case where they ignore uncertainty at both the individual and the system level.
\end{abstract}

\section{INTRODUCTION}

Queuing research has a wide gamut of applications spanning disciplines, which include telecommunications, informatics, economics, mathematics etc. Queuing theory is the mathematical analysis of queues (waiting lines) and the derivation of its performance measures (e.g. average waiting time). Erlang (Erlang 1909) the Danish engineer, who published his work on telephone traffic problem in 1909, is considered to be the father of queuing theory (Gross and Harris 1998).

Since then queuing has been studied extensively and tackled mainly with an aggregated view on the processes involved. However, queuing is a dynamic process where customers' decisions (disaggregated view) depend on the state of the system under study. Koole and Mandelbaum (2002) emphasize the need for including human factors in the context of call centre queuing models. Since Naor (1969) and Yechiali (1969) published their seminal papers, some researchers have tried to shift from the traditional approach, based on performance measures, to an approach wherein customers' decisions are incorporated into the queuing model.

Customers' decisions in queuing systems with stochastic arrival and service patterns have been studied

\author{
Karthik Sankaranarayanan and Erik R. Larsen \\ Institute of Management \\ University of Lugano \\ 6904, Lugano, Switzerland \\ E-mail: erik.larsen@usi.ch, \\ karthik.sankaranarayanan@usi.ch
}

theoretically in operations research and management science by Edelson and Hilderbrand (1975), Stidham (1985), Dewan and Medelson (1990), van Ackere et al. (1995), Rump and Stidham (1998), Zohar et al. (2002), among others. Hassin and Haviv (2003) discuss a vast literature, which depicts widely a framework on equilibrium behaviour in stochastic queuing systems. Comparatively, deterministic models have not been much discussed. Some models were proposed by Edelson (1971), Agnew (1976), Haxholdt et al. (2003), van Ackere and Larsen (2004), van Ackere et al. (2006).

Haxholdt et al. (2003) and van Ackere et al. (2006), using system dynamics, have included feedback into their model to look at the return rates of customers to the service facility. van Ackere and Larsen (2004) have used a one-dimensional cellular automata (CA) model in order to study formation of customers' expectations about congestion on a three-road system. Sankaranarayanan et al (2010a, 2010b) and Delgado et al. (2011) have applied this approach for a multichannel service facility with similar service rates for all facilities. They use an agent-based modelling (ABM) approach to understand and analyze how customers adapt their decisions based on adaptive expectations (Nerlove 1958). How local interactions among customers influence the formation of queues and how this formation is depicted by different collective behaviours, are explained. They use genetic algorithms to optimize the behavioural parameters of the agent-based model.

In this paper we propose an extension of the model of Sankaranarayanan et al. (2010a) and Delgado et al. (2011). We model a queuing system with endogenous and deterministic arrival rates using an agent-based simulation approach, more precisely a one-dimensional cellular automata, in order to study the collective behaviour of customers (referred to as agents in the remainder of the paper in order to contextualize the problem to the ABM methodology ) who must choose routinely a facility for service. Some examples of this kind of systems include: a person who goes weekly to the supermarket, a person who must choose a garage for the inspection of her car, and a person who goes monthly to the bank to pay her bills.

We introduce uncertainty into the process of formation of agents' expectations in order to analyse how a risk- 
averse attitude may affect collective behaviour. In this way we differ from Sankaranarayanan et al. (2010a) and Delgado et al. (2011) since the agents' decision policy in our model considers both the agents expectations and their uncertainty regarding those expectations. In order to model the agents' uncertainty we use the concept of volatility of forecast errors (e.g. Taylor 2004, 2006).

Our simulation results indicate that the transient period of the system is longer when its agents have an intermediate degree of risk aversion $(R=0.5)$, than when they are risk-neutral. After this transient period, if agents are risk-averse, the system converges more slowly to an almost-stable average sojourn time. Additionally, risk-agents are more likely to "forget" a facility, thus having a lower probability to be close to the Nash equilibrium (which requires using all facilities) when a steady state is reached. Systems where agents are either risk-neutral or strongly risk-averse perform better than those who have an intermediate level of risk aversion.

This paper is organized as follows: in the next section we describe the queuing system, the concepts of expectations and uncertainty, and how we use agentbased simulation and adaptive expectations to model the problem. In the third section we discuss and explain the simulation results. Conclusions and future work are presented in the last section.

\section{MODEL DESCRIPTION}

As pointed out earlier this paper builds on the work of Sankaranarayanan et al. (2010a) and Delgado et al. (2011); hence we briefly explain their model and then elaborate on how we incorporate the concept of uncertainty into the model.

Consider a fixed population of $\mathrm{n}$ customers (referred to as agents) who must routinely patronize a facility for service. Each period they face the decision of choosing among three facilities for this service. Each facility is set up with a queue. Queues are unobservable (Hassin and Haviv 2003), i.e. agents do not know the state of the system. Their decision therefore depends on their expectations about system congestion. The service rate $(\mu)$ is assumed to be fixed and identical for all facilities. The arrival rate is endogenous and depends on the agents' decisions. Reneging and balking are not allowed.

We use agent-based simulation to model this system; more precisely a cellular automata approach (Gutowitz 1991, Wolfram 1994) is adopted to model the interaction between agents, capture their expectations and analyse their collective behaviour. Agents interact in a one-dimensional neighbourhood assumed to have the shape of a ring, where one neighbour is located to the left and right of each agent. The last agent has to the first one and the last but one as neighbours. As we simulate a system with just three facilities, considering a neighbourhood size larger than 1 would be to assume that customers could often have full information. The neighbourhood can be assumed to represent, for instance, a social network encompassing colleagues, friends, people living next-door etc.

Each time period agents must choose a queue (state) following a decision rule, which is based on their expectations of sojourn time for the different queues and their uncertainty regarding these expectations. We assume smart agents who have the ability to remember information and update their memory using new information. This memory is updated using an adaptive expectations model (Nerlove 1958). Agents form expectations based on their last experience and that of their best performing neighbour, i.e. the neighbour who has experienced the minimum sojourn time in the previous period. Then, agents' expectations are adjusted using their uncertainty regarding such expectations. We model this uncertainty by applying the concept of volatility of forecasting errors (e.g. Taylor 2004). As variance is unobservable, exponential smoothing can be applied to estimate the squared residuals (Taylor 2006). Technically the model works in the following way.

Let $A$ be a set of $n$ agents (cells) $\left\{A_{1}, A_{2}, \ldots, A_{i}, \ldots, A_{n}\right\}$ interacting with their neighbours to select a facility among a set of $m$ facilities (states) $\left\{Q_{l}, Q_{2}, \ldots, Q_{j}, \ldots\right.$, $\left.Q_{m}\right\}$ for service. The agents' decisions determine their state (queue) for the next period and this state will depend on their expected sojourn time for each facility, their uncertainty and how risk-averse they are. We denote expectations by $M_{i j t}$ and uncertainty by $s_{i j t}$. $R$ is a "risk aversion factor" which is identical for all agents. Let $S M_{i j t}$ be an adjusted measure for the expected sojourn time of agent $A_{i}$ at facility $Q_{j}$ at time $t$. We call this measure "adjusted" because it incorporates the agents' uncertainty and their degree of risk aversion. The more risk-averse they are, the larger $R$ is. $S M_{i j t}$ is determined as follows:

$$
S M_{i j t}=M_{i j t}+R^{*} s_{i j t}
$$

$S M_{i j t}$ can be interpreted as a form an upper bound of the agents' expected sojourn time. The agents' uncertainty, $s_{i j t}$, is assumed to be the volatility of their expectations and the risk aversion factor, $R$, may be considered as the how sensitive agents are to this volatility. In order to decide which facility to join, agents update this measure each period, based on the updated values of $M_{i j t}$ and $s_{i j t}$ as explained below.

\section{Estimating the Expected Sojourn Times}

The expectation $M_{i j t}$ is updated using an exponential smoothing process, also called adaptive or exponential forecasting (e.g. Nerlove 1958; Theil and Wage 1964; Gardner Jr. 2006). It is a mathematical-statistical method of forecasting commonly applied to financial 
market and economic data, but it can be used with any discrete set of repeated measurements (Gardner Jr. 2006). This technique is based on weighted averages of two sources of evidence: The latest evidence (the most recent observation), and the value computed one period before (Theil and Wage, 1964). Exponential smoothing allows estimating the expected sojourn time, $M_{i j t+1}$, at time $t+1$ as a weighted average of the previous estimation of the expectation, $M_{i j t}$, and the recent observation $W_{i j t}$ (experience). According to this, agents update their expectations for their chosen queue and that of their quickest neighbour using an exponentially weighted average with weight $\alpha$. So, $M_{i j t}$ can be expressed by:

$$
M_{i j t+1}=\alpha M_{i j t}+(1-\alpha) W_{i j t}
$$

where $\alpha$ is also known as the coefficient of expectations (Nerlove 1958) or smoothing parameter (Gardner Jr. 2006). See Delgado et al. 2011 for more technical details about the estimation of $M_{i j t}$.

\section{Estimating the Uncertainty Measure for the Agents' Expected Sojourn Times}

The measure of uncertainty, $s_{i j t}$, is modelled using the error of the estimation of $M_{i j t}$. According to Newbold (1988), if $M_{i j t}$ is a smoothing estimation of $W_{i j t}$, the error in such an estimation will be:

$$
e_{i j t}=W_{i j t}-M_{i j t-1}
$$

and the cumulative error on all expectations at time $t$ could be estimated by the sum of the squared errors:

$$
S S=\sum_{t=2}^{t s i m} e_{t}^{2}=\sum_{t=2}^{t s i m}\left(W_{i j t}-M_{i j t-1}\right)^{2}
$$

where $t$ sim is the simulation time. However, in this way all the observations are given the same weight; a more realistic approach is to give a different weight to more recent errors. So in this context, the uncertainty, $s_{i j t}$, may be estimated using the concept of volatility forecasting which is calculated by means of the smoothing variance (e.g. Taylor 2006). As variance is unobservable, we can apply exponential smoothing in order to estimate this variance using the squared residuals (Taylor 2004). Thus, the smoothed variance, $s_{i j t+1}^{2}$, will be expressed as a weighted average of the previous estimate, $s_{i j t}^{2}$, and the new observation of the squared estimate error $e_{i j t}^{2}$. Thus, agents update the variance, $s_{i j t}^{2}$, as follows:

$$
s_{i j t+1}^{2}=\gamma * s_{i j t}^{2}+(1-\gamma) *\left(W_{i j t}-M_{i j t-1}\right)^{2}
$$

where $\gamma$ is the smoothing parameter (Taylor 2004). the volatility is then measured by the standard deviation $s_{i j t}$.

Once the uncertainty is estimated, agents consider this value in order to estimate an upper bound of their expected sojourn time for each facility. They then decide which facility to join the next period based on this adjusted estimate. Regarding the decision rule, we assume rational agents who always patronize the facility with the lowest upper bound for the expected sojourn time $\left(S M_{i j t}\right)$, i.e. agents update their state by choosing the queue with the lowest value of $S M_{i j t}$ in Equation (1). In the rare case where two or more queues have the same minimal adjusted expected sojourn time, agents choose among these facilities, giving first preference to their previously chosen queue and second choice to the one previously used by their best performing neighbour.

\section{Average Sojourn Time in a Transient State}

In this paper we consider a queuing system whose arrival rates may temporarily exceed the service rates. Hence we need a measure for the average sojourn time that enables us to study the system behaviour in a transient state, rather than in steady state

This measure is proposed and explained in Sankaranarayanan et al. (2010a). They consider a congestion measure which satisfies the well known Little's Law and the steady state equations (Gross and Harris 1998), but remains well-defined when $\rho \geq 1$ (Transient Analysis). Such a measure is given by:

$$
W_{j t}=\frac{\lambda_{j t}}{\mu^{2}}+\frac{1}{\mu}
$$

where $\mu$ is the service rate for all facilities and $\lambda_{j t}$ the number of agents arriving at $Q_{j}$ at time $t$. For more details about the formulation of this measure, see Sankaranarayanan et al. (2010a) and Delgado et al. (2011). This measure is used throughout the paper.

\section{SIMULATION RESULTS AND DISCUSSION}

In order to study the impact of including uncertainty on the agents' decisions, we divide the simulation results in three parts: first we simulate a run of our chosen base case, which is described in Table 1. Using this simulation we compare the collective behaviour resulting from decisions, which include or ignore uncertainty. These results are reported in Figures 1 to 4 . Then we study the distribution of average sojourn time for different values of the risk aversion factor, $R$, while keeping constant the smoothing parameters (Figure 5). Finally we perform a sensitivity analysis by considering combinations of smoothing parameters $\alpha$ and $\gamma$ (Figure 6). 
Table 1: Parameter values used for the simulation runs

\begin{tabular}{|c|c|c|}
\hline Parameter & Value & Description \\
\hline $\mathrm{m}$ & 3 & Number of service facilities \\
\hline $\mathrm{n}$ & 120 & Population size \\
\hline$\mu$ & 5 & Service rate \\
\hline$\alpha$ & 0.3 & $\begin{array}{l}\text { Weight to memory when updating } \\
\text { the expected sojourn time }\end{array}$ \\
\hline$\gamma$ & 0.7 & $\begin{array}{l}\text { Weight to memory when updating } \\
\text { the estimated variance of the } \\
\text { expected sojourn time }\end{array}$ \\
\hline Tsim & 100 & Simulation time \\
\hline
\end{tabular}

Figures 1 and 2 exhibit the evolution of the agents' choices of service facility over 100 time periods for the same initial values of expected sojourn times allocated, which were allocated randomly to the agents. The horizontal axis depicts the time, while the 120 agents are represented on the vertical axis from top to bottom. The colours indicate the state (the chosen facility) of each agent each period. Black indicates facility 1, gray facility 2 and white facility 3 .

Both figures exhibit an initial transient period. This period is longer when agents consider uncertainty in their decisions (around 34 periods, see Figure 2) compared to the case where uncertainty is ignored (around 15 periods, see Figure 1). This length can also vary depending on the randomly allocated initial expected sojourn times (Delgado et al. 2011). During this period agents explore all facilities in order to capture information about each one and try to learn from the system behaviour. The weight they give this information leads to herding: agents tend to imitate their best performing neighbours and at the end of the transient period one or two facilities tends to be crowded, as shown in Figure 1 (between periods 9 and 15 ) and Figure 2 (between periods 27 and 34). As more weight is given to new information than to memory ( $\alpha$ is less than 0.5), agents react to the bad experiences by changing facility at the next period. For instance, in Figure 1 facility 1 is crowded at time 10, implying that no agents choose this facility in the next two periods. A similar situation occurs in Figure 2 at time 30 when all agents join facility 3 and it will not be used for the next three periods.

After the transient period a collective behaviour pattern starts to emerge. Small groups of agents tend to stay at the same facility over time, resulting in a certain degree of stability for the system. However the agents located on the borders between these groups keep shifting between two facilities (e.g. in Figure 1 agents 11 and 21 switch between facilities 1 (black) and 3 (white)). Between periods 15 and 65 agents on the borders change between facilities irregularly. After period 65 agents reach a regular pattern, which can be considered as stable. Comparatively, this behaviour cannot be identified in figure 2; at time 100, agents have not yet reached such a form of stability.

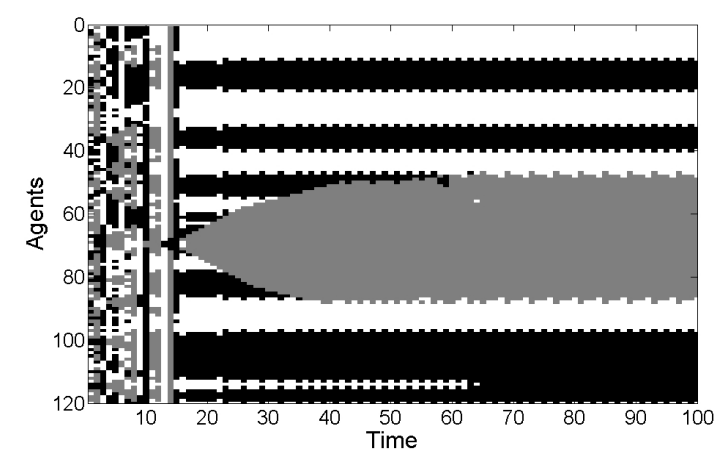

Figure 1. Spatial-temporal behavioural evolution of agents' choice of service facility with $\alpha=0.3 ; \gamma=0.7$ and $\mathrm{R}=0$

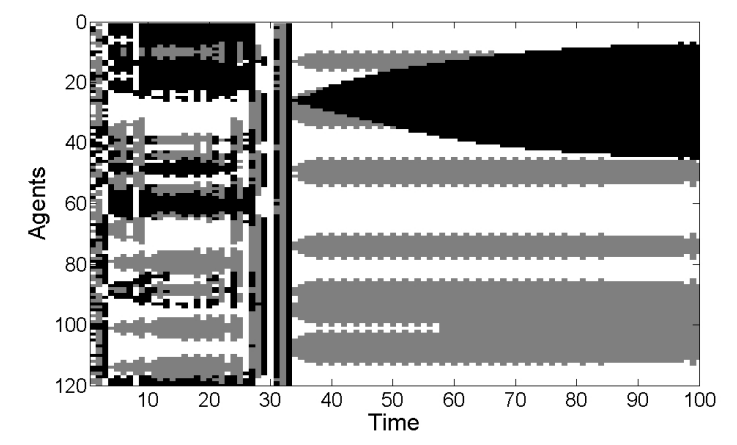

Figure 2. Spatial-temporal behavioural evolution of agents' choice of service facility with $\alpha=0.3 ; \gamma=0.7$ and $\mathrm{R}=0.5$

In both cases, two facilities have several small groups of loyal agents (facility 1 and 3 in Figure 1 and facility 2 and 3 in Figure 2), while one big group is loyal to the third facility (facility 2 in Figure 1 and facility 1 in figure 2). In Figure 1 this big group emerges at time 16 because when facility 2 reached its maximum level of congestion two periods earlier (at time 14), two agents did not use this facility. Their memory was therefore not affected by this bad performance, while that of the other agents increased a lot. At time 15 these two agents stayed at facility 1 , which was then the most visited. Consequently their expectation for facility 1 soared. Thus at time 16, these two agents were the only ones to join facility 2 , all other agents considering it a very poor choice. Hence, in this period of time they experienced an extremely low sojourn time as shown in Figure 3. They shared this experience with their neighbours over the next periods, who started coming back to facility 2 . This increases the number of agents patronizing this facility, and thus the sojourn time and the agents expected sojourn time. At some point (around period 42) the last agents to join are disappointed and, given the information from their neighbours (who did not yet join), they leave again. These agents continue to alternate irregularly between this facility and that of 
their best performing neighbour until period 65. After this period the switching pattern stabilizes as discussed above. Agent 56 illustrates an odd case at time 64: facility 2 being close to its maximum occupation, the expected sojourn time of agent 56 for this facility increases and the minimum expected sojourn time of agent 56 at time 64 switches to facility 3 (in white). However, this agent experiences a bad sojourn time, which encourages him to come back to facility 2 and stay there for the remainder of the simulation.

A similar behaviour occurs in Figure 2, but the system requires more time to stabilize: no regular pattern is reached by the end of the simulation.

In Figures 3 and 4 we can observe that in those periods when most agents patronize the same facility the maximum, minimum and average sojourn time experienced by the agents reach extreme values. For instance, when agents ignore uncertainty (Figures 1 and 3 ) most agents choose facility 3 at time 13, two agents facility 1 and no agents facility 2 . Hence, the two agents at facility 1 experience the lowest sojourn time (min $\left.\left(W_{j, t}\right)=W_{l, 14}=0.28\right)$, while the agents at facility 3 experience a very high sojourn time $\left(W_{3,14}=4.92\right)$. A similar case occurs in Figures 2 and 4, at time 34, a single agent joins facility 1 and experience the lowest sojourn time $\left(\min \left(W_{j, t}\right)=W_{1,34}=0.24\right)$.

Figures 3 and 4 also illustrate that a system in which agents are risk-neutral converges faster to an almoststable average sojourn time than a system with riskaverse agents.

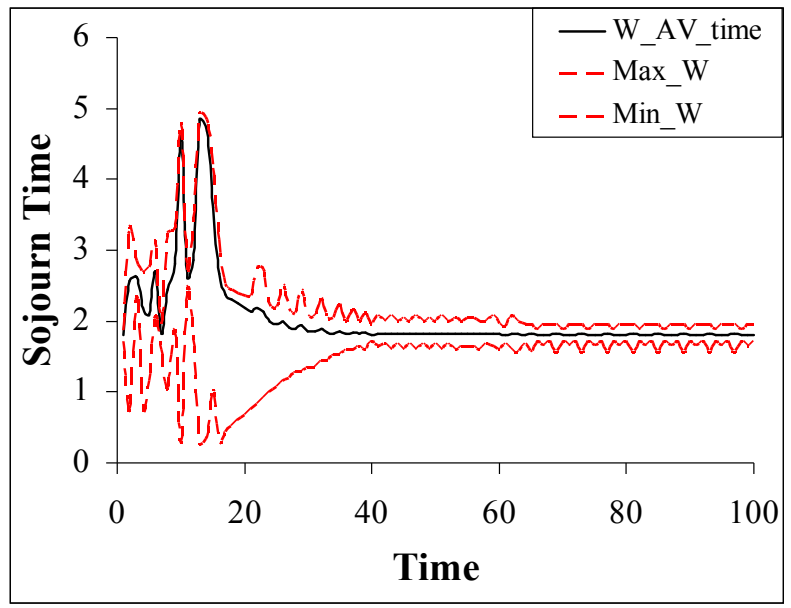

Figure 3. Average, maximum and minimum sojourn time for parameters $\alpha=0.3 ; \gamma=0.7$ and $\mathrm{R}=0$ (Without uncertainty)

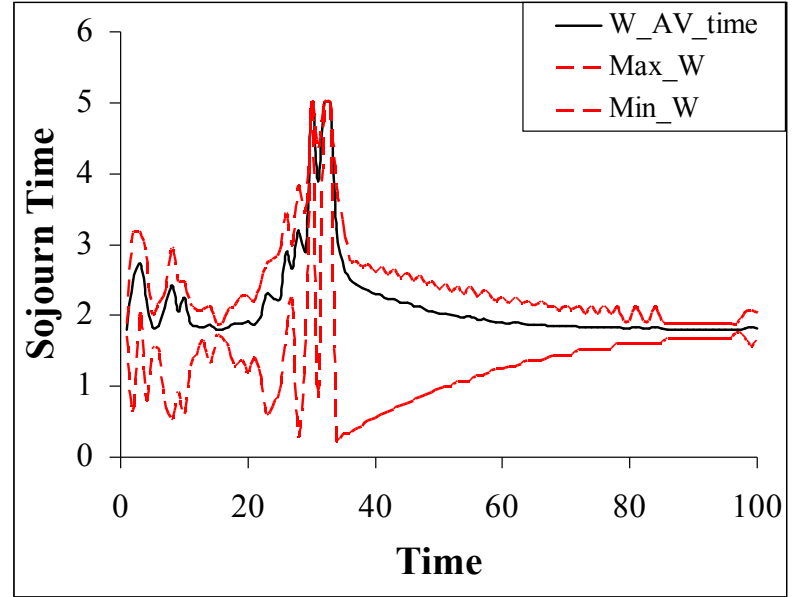

Figure 4. Average, maximum and minimum sojourn time for parameters $\alpha=0.3 ; \gamma=0.7$ and $\mathrm{R}=0.5$

Figure 5 shows the distributions of the average sojourn time for 1000 simulations of a system configured according to Table 1 with $R$ equals to 0 (uncertainty is ignored) and 0.5 (a certain risk-averse attitude is considered). Each simulation was run over 1500 time periods and different initial expected sojourn time allocated to the agents randomly. The average sojourn time was calculated based on the last 500 periods of each run.

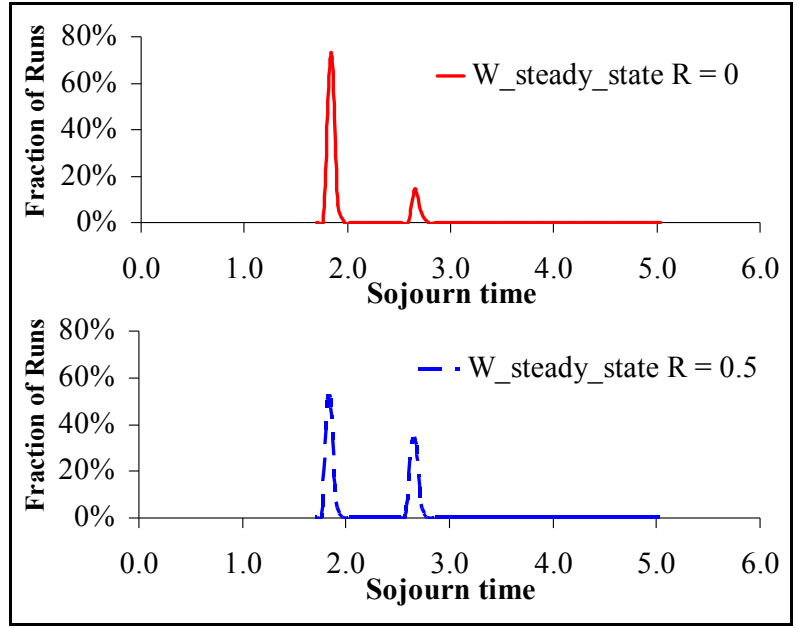

Figure 5. Distributions of average sojourn time for 1000 simulation with different initial conditions for $\alpha=0.3 ; \gamma$ $=0.7$

Figure 5 illustrates the dependence of the sojourn time on the initial allocated expected sojourn times and enables us to identify the probability that a queue will be ignored by the agents once the system reaches a steady state. The continuous line depicts the distribution of average sojourn times for the system where agents ignore uncertainty and the dashed one the system in which agents incorporate uncertainty into their decisions. The first peak of both distributions represents the proportion of cases where the three facilities are 
used in steady state. When $R=0$, around $81 \%$ of the cases reach an average sojourn time in steady-state between 1.80 and 1.90 time units, compared to $57 \%$ of the cases for $R=0.5$. The remaining cases (19\% for $R=$ 0 and $43 \%$ for $R=0.5$ ) fall in the interval [2.58, 2.8]. In these cases one facility is ignored when the system has reached a steady state, i.e. all the agents are clustered in only 2 of the 3 facilities.

Figure 6 exhibits a sensitivity analysis. We consider several combinations of smoothing parameters $\alpha$ and $\gamma$, while varying the risk aversion factor from 0 to 1.5 in increments of 0.1 . We simulate 1000 iterations for each combination of parameters $(\alpha, \gamma, R)$. Each iteration corresponds to a different set of initial expected sojourn times allocated to the agents randomly. All parameter combinations use the same random seeds in order to avoid a biased comparison.

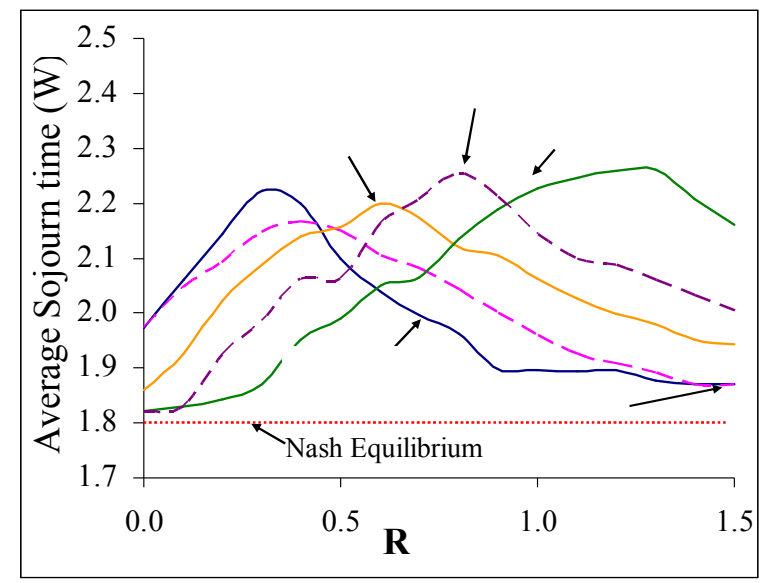

Figure 6. The Average Sojourn time in steady state as a function of $\mathrm{R}$ for selected values of $\alpha$ and $\gamma$. Each value represents the average of 1000 simulations.

Given that the three facilities have the same service capacity, the Nash equilibrium of the system is only achieved when agents are split equally across the three facilities. Such an equilibrium yields a sojourn time of 1.8 time units. Figures 6 indicates that on average the agents perform above the Nash equilibrium.

Figure 6 illustrates that when agents ignore uncertainty, that is $R=0$, they perform better if they give at least the same weight to their memories as to the new information, i.e. if $\alpha \geq 0.5$. On the contrary, if $\alpha<0.5$, very risk-averse agents (i.e. $R$ is highest) perform better. That is, risk-neutral agents who are more conservative regarding new information (their experience and their neighbour's experience) achieve lower soujourn times on average than agents who give more weight to recent information. Likewise, agents attaching more importance to new information and having higher riskaversion levels achieve sojourn times similar to those of conservative risk-neutral agents.
Next consider the parameter, $\gamma$, used in the variance smoothing process. Risk-averse agents must assign a high value to this parameter, i.e. $\gamma \geq 0.5$, if they want to perform well; otherwise they must use values $\alpha$ less than 0.5 .

If agents use the same value for both smoothing parameters, those who are very risk-averse reach a better performance when they give more weight to new information, i.e. $\alpha$ and $\gamma$ are both low.

In general terms, agents with an intermediate riskaversion tend to perform more poorly than those who are either very risk-averse or close to being risk-neutral.

\section{CONCLUSIONS AND FUTURE WORK}

This paper uses a CA model to study the behavioural aspects involved in a queuing system in which customers decide what facility to choose for service each time period. They are endowed with memory and characterized by their risk attitude. They base their decisions on their expectation of the sojourn times and their uncertainty regarding these expectations.

Risk-neutral agents base their decision on the expected sojourn time, while risk-averse agents estimate an upper bound for the different sojourn times. When agents have an intermediate degree of risk aversion $(R=0.5)$, the system exhibits a longer transient period and, after this transient period, the system converges more slowly to an almost-stable average sojourn time. Additionally, riskaverse agents are more likely to "forget" a facility, thus having a lower probability to be close to the Nash equilibrium (which requires using all facilities) when a steady state is reached. Systems where agents are either close to risk-neutral or strongly risk-averse perform better than those who have an intermediate level of risk aversion.

The optimal choice of updating parameters depends on the agents' risk attitude. Consequently, future research will focus on studying the impact of the different behavioral parameters $(\alpha, \gamma, R)$. This will include giving different weights to own experience and information received from neighbors in the memory updating processes (expected sojourn times and variance). The next step will be to assume heterogeneous agents, i.e. agents with different smoothing parameters and risk factors in the same system. Finally, we plan to study the interaction between the decisions of managers and customers by allowing for facilities with different, adjustable service capacities.

\section{REFERENCES}

Agnew, C. E., 1976. Dynamic modelling and control of congestion-prone systems. Operations Research 24, No. 3, 400-419. 
Delgado, C.A.; van Ackere, C.A.; Larsen, E.R.; and K. Sankaranarayanan. 2011. "Collective Behavioral Patterns in a Multichannel Service Facilities System: a Cellular Automata Approach". In Proceeding of the $12^{\text {th }}$ INFORMS Computing Society Conference, ICS 2011 (Monterey, CA. Jan. 9- 11). INFORMS, Hanover, MD, 16-28.

Dewan, S. and Mendelson, H., 1990. User delay costs and internal pricing for a service facility. Management Science 36, No. 12, 1502-1517.

Edelson, N. M.,1971. Congestion Tolls under Monopoly. American Economic Review 61, No. 5, 873-882.

Edelson, N. M. and Hildebrand, D. K., 1975. Congestion Tolls for Poisson Queuing Processes. Econometrica 43, No. 1, 81-92.

Erlang, A.K. 1909. The theory of probabilities and telephone conversations. Matematisk Tidsskrift 20(B), 33-39.

Gardner Jr., E.S. 2006. Exponential smoothing: The state of the art - Part II. International Journal of Forecasting 22, No. 4(Oct-Dec), 637-666.

Gross, D. and Harris, C.M. 1998. Fundamentals of Queueing Theory. Wiley, New York. 3rd edition.

Gutowitz, H. 1991. Cellular Automata: Theory and Experiment. North-Holland, MIT Press, Boston, MA.

Hassin, R. and Haviv, M. 2003. To Queue or Not to Queue: Equilibrium behavior in Queueing systems. Kluwer, Boston, MA.

Haxholdt, C.; Larsen, E.R.; and A. van Ackere. 2003. Mode locking and chaos in a deterministic queueing model with feedback. Management Science 49, No. 6, 816-830.

Koole, G. and Mandelbaum, A. 2002. Queueing models of call centers: An introduction. Annals of Operations Research 113, No. 1, 41-59.

Naor, P. 1969. On the regulation of queue size by levying tolls. Econometrica 36, No. 1, 15-24.

Nerlove M., 1958. Adaptive expectations and Cobweb phenomena. The Quarterly Journal of Economics 72, No. 2, 227-240.

Newbold, P. 1988. Statistics for Business and Economics. Prentice Hall, Englewood Cliffs, N.J. $2^{\text {nd }}$ ed.

Rump, C. M. and Stidham, S. Jr. (1998). Stability and chaos in input pricing for a service facility with adaptive customer response to congestion. Management Science 44, No. 2, 246-261.

Sankaranarayanan, K.; Delgado, C.A.; van Ackere, A.; and E. R. Larsen. 2010a. "The Micro-Dynamics of queuing understanding the formation of queues". Working paper, Institute of Management, University of Lugano.

Sankaranarayanan, K.; Larsen, E.R.; van Ackere, A.; and Delgado, C.A. 2010b. "Genetic Algorithm based Optimization of an Agent Based Queuing System". In Proceedings of the IEEE Industrial Engineering and Engineering Management Conference 2010, (Dec. 7-10 Macau). IEEEXplore ${ }^{\circledR}$ Digital Library, 1344-1348.

Stidham, S., 1985. Optimal control of admission to a queueing system. IEEE Trans. Automatic Control, AC 30, 705-713.

Taylor, J.W. 2004. "Volatility forecasting with smooth transition exponential smoothing". International Journal of Forecasting 20, No. 2(Apr-Jun), 273-286.

Taylor, J.W. 2006. "Invited Comments on "Exponential Smoothing: The state of the Art - Part II" by E.S. Gardner, Jr." International Journal of Forecasting 22, No. 4(Oct-Dec), 671-672.

Theil, H. and Wage, H. 1964. Some observations on adaptive forecasting. Management Science 10, No. 2, 198-206. van Ackere, A., 1995. Capacity management: pricing strategy, performance and the role of information. Int. J. Production Economics 40, No 1, 89-100

van Ackere, A. and Larsen, E.R. 2004. Self-organizing behavior in the presence of negative externalities: A conceptual model of commuter choice. European Journal of Operational Research 15, No. 2, 501-513

van Ackere, A.; Haxholdt, C.; and E.R. Larsen. 2006. Long and short term customer reaction: a two-stage queueing approach. System Dynamics Review 22, No. 4, 349-369.

Wolfram, S. 1994. Cellular automata and complexity. Westview Press, Champaign, IL.

Yechiali, U. 1969. On optimal balking rules and toll charges in the GI/M/1 queuing process. Operations Research 19 , No. 2, 349-370.

Zohar, E. Mandelbaum, A. and N. Shimkin. 2002. Adaptive behavior of impatient customers in tele-queues: theory and empirical support. Management Science 48, No. 4, 566583

\section{AUTHOR BIOGRAPHIES}

CARLOS A. DELGADO A. is a PhD student in Business Information Systems and teaching assistant at HEC Lausanne, the School of Business and Economics of the University of Lausanne, Switzerland. He received his Masters in Systems Engineering and a Bachelor of Industrial Engineering from the Faculty of Mines, National University of Colombia, where he also worked for three years as a Research Engineer and Teaching Assistant for the Bachelor course in systems simulation.

ANN VAN ACKERE is Professor of Decision Sciences at HEC Lausanne, the School of Business and Economics of the University of Lausanne, Switzerland since 1999. She obtained her PhD from the Stanford Graduate School of Business and joined the faculty of London Business School, UK, upon graduation.

KARTHIK SANKARANARAYANAN is a PhD student and research assistant at the Institute of Management, University of Lugano, Switzerland. He has a Masters in Embedded Systems Design and Management and a Bachelors degree in Electrical and Electronics Engineering. Previously he worked as a researcher at CEDT, Indian Institute of Science, Bangalore, India.

ERIK R. LARSEN is Professor at the Institute of Management and vice dean of the Faculty of Economics at the University of Lugano, Switzerland. Previously he held appointments at Cass Business School (London), London Business School, and Copenhagen Business School. During the period 1996-1998, he was an EU Marie Curie Fellow at the University of Bologna, Italy. He obtained his PhD from the Institute of Economics, Copenhagen Business School and his M.Sc. from the Technical University of Denmark. 